\title{
Schlafmedizinische Forschung in Deutschland
}

\section{Sleep Research in Germany}

Autor

Institut

\section{H.-W. Duchna}

Fachkliniken Wangen, Waldburg-Zeil Kliniken, Medizinische Klinik für Atemwegserkrankungen und Allergien

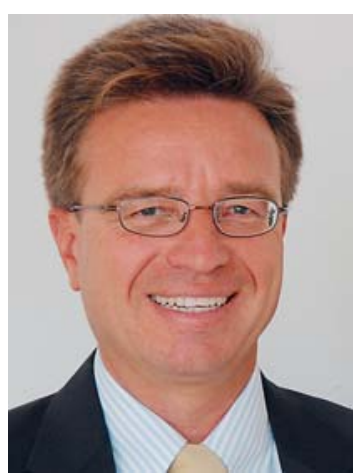

Prof. Dr. med. H.-W. Duchna
Die Schlafmedizin hat in Deutschland mittlerweile einen festen Platz innerhalb der Inneren Medizin und insbesondere der Pneumologie eingenommen, wobei starke Schnittstellen mit den kardiovaskulären Erkrankungen, chronischen Atemwegserkrankungen, Diabetes mellitus, Adipositas, Nierenerkrankungen, Schmerzsyndromen, aber auch neurologischen Erkrankungen bis hin zu Fragen hinsichtlich einer Fahrtauglichkeit bestehen. Zunehmend werden komplexe schlafbezogene Atmungsstörungen, Kombinationen aus obstruktiven und zentralen schlafbezogenen Atmungsstörungen, aber auch HypoventilationsSyndrome identifiziert, die entsprechend vielfältige und teilweise auch komplizierte Therapieverfahren bedingen. Die Mitglieder der Sektion Schlafmedizin (Sektion 8) der Deutschen Gesellschaft für Pneumologie und Beatmungsmedizin
(DGP) konzentrieren sich in enger Kooperation mit der Deutschen Gesellschaft für Schlafforschung und Schlafmedizin (DGSM) sowie Vertretern der Deutschen Gesellschaft für Kardiologie (DGK) auf die klinische Versorgung der Patienten mit Schlafstörungen bzw. schlafbezogenen Atmungsstörungen, gleichzeitig treiben aber auch viele Kolleginnen und Kollegen die medizinisch wissenschaftlichen Aktivitäten auf dem Gebiet der Schlafmedizin gerade in Deutschland voran. Herr Prof. Randerath (Sektionssprecher) und ich (stellv. Sprecher) möchten den Lesern der Zeitschrift Pneumologie mit der Serie „Schlafmedizinische Forschung in Deutschland" sowohl aktuelle Trends und Schwerpunktthemen innerhalb der Sektion 8 als auch „wissenschaftliche Keimzentren“ auf dem Gebiet der Schlafmedizin in Deutschland präsentieren.

Bibliografie

DOI http://dx.doi.org/

10.1055/s-0032-1309413

Pneumologie 2012; 66: 463

(c) Georg Thieme Verlag KG

Stuttgart · New York

ISSN 0934-8387

Korrespondenzadresse

Prof. Dr. med.

Hans-Werner Duchna

Fachkliniken Wangen,

Waldburg-Zeil Kliniken

Medizinische Klinik für

Atemwegserkrankungen und

Allergien

Am Vogelherd 14

88239 Wangen

hans-werner.duchna@

wz-kliniken.de 